\title{
Neuroadrenergic and reflex abnormalities in patients with metabolic syndrome
}

Received: 20 December 2004 / Accepted: 7 March 2005 / Published online: 3 June 2005

(C) Springer-Verlag 2005

\begin{abstract}
Aims/hypothesis: Previous studies have shown that alterations in vascular, metabolic, inflammatory and haemocoagulative functions characterise the metabolic syndrome. Whether this is also the case for sympathetic function is not clear. We therefore aimed to clarify this issue and to determine whether metabolic or reflex mechanisms might be responsible for the possible adrenergic dysfunction. Methods: In 43 healthy control subjects (age $48.2 \pm 1.0$ years, mean \pm SEM) and in 48 untreated agematched subjects with metabolic syndrome (National Cholesterol Education Program's Adult Treatment Panel III Report criteria) we measured, along with anthropometric and metabolic variables, blood pressure (Finapres), heart rate (ECG) and efferent postganglionic muscle sympathetic nerve activity (microneurography) at rest and during baroreceptor manipulation (vasoactive drug infusion technique). Results: Compared with control subjects, subjects with metabolic syndrome had higher BMI, waist circumference, blood pressure, cholesterol, triglycerides, insulin and homeostasis model assessment (HOMA)
\end{abstract}

G. Grassi · R. Dell'Oro · F. Quarti-Trevano · F. Scopelliti ·

F. Paleari · P. L. Gamba · G. Mancia

Department of Internal Medicine,

Prevention and Medicine Biotechnologies,

University Milano-Bicocca,

Milan, Italy

G. Grassi · G. Mancia

Clinical Physiology and Hypertension Center,

IRCCS Maggiore Hospital,

Milan, Italy

G. Grassi · G. Seravalle · G. Mancia

IRCCS Istituto Auxologico Italiano,

Milan, Italy

G. Mancia $(\square)$

Clinica Medica, Ospedale S. Gerardo,

Via Donizetti 106 ,

20052 Monza, Milano, Italy

e-mail: giuseppe.mancia@unimib.it

Tel.: +39-039-2333357

Fax: +39-039-322274 index values but lower HDL cholesterol values. Sympathetic nerve traffic was significantly greater in subjects with metabolic syndrome than in control subjects $(61.1 \pm$ 2.6 vs $43.8 \pm 2.8$ bursts $/ 100$ heartbeats, $p<0.01$ ), the presence of sympathetic activation also being detectable when the metabolic syndrome did not include hypertension as a component. Muscle sympathetic nerve traffic correlated directly and significantly with waist circumference ( $r=$ $0.46, p<0.001)$ and HOMA index $(r=0.49, p<0.001)$ and was inversely related to baroreflex sensitivity $(r=-0.44$, $p<0.001$, which was impaired in the metabolic syndrome. Conclusions/interpretation: These data provide evidence that the metabolic syndrome is characterised by sympathetic activation and that this abnormality (1) is also detectable when blood pressure is normal and (2) depends on insulin resistance as well as on reflex alterations.

Keywords Baroreceptor - Insulin · Metabolic syndrome · Obesity $\cdot$ Sympathetic nervous system

Abbreviations HOMA: Homeostasis model assessment

\section{Introduction}

The metabolic syndrome carries an increase in cardiovascular risk [1-3] and its occurrence is associated with alterations in a spectrum of cardiovascular functions, such as endothelial impairment, a proinflammatory state, an alteration in oxidative stress and a dysfunction in the haemocoagulative process $[4,5]$. The alterations might include sympathetic hyperactivity, because the metabolic syndrome is associated with insulin resistance and hyperinsulinaemia, which has sympathostimulating effects $[6,7]$. This has not been established conclusively, however, because the only study [8] so far that has directly assessed sympathetic activity in the metabolic syndrome was performed in patients treated with cardiovascular drugs known to affect the metabolic variables on which diagnosis of the disease is based $[9,10]$ and to consistently modify adrenergic cardiovascular drive [9-11]. 
We set out to address this issue by directly quantifying efferent postganglionic sympathetic nerve traffic by using the microneurographic technique $[11,12]$ in untreated patients fitting the criteria for the diagnosis of metabolic syndrome issued by the National Cholesterol Education Program's Adult Treatment Panel III Report (ATP III) [13]. The study was also designed to (1) determine whether, in this condition, sympathetic activation was dependent on hypertension as a component of the metabolic syndrome, and (2) provide information on the metabolic and reflex mechanisms underlying the possible adrenergic dysfunction.

\section{Subjects and methods}

Study population The study population consisted of subjects of both sexes with an age ranging from 36 to 54 years, who were included if they had (1) no secondary hypertension, (2) no atrial fibrillation or other major cardiac arrhythmias, (3) no history of myocardial infarction or clinical or laboratory evidence of valvular heart disease, (4) no history of smoking and/or excessive alcohol consumption, (5) no history or clinical evidence of congestive heart failure, renal insufficiency or major non-cardiovascular diseases, and (6) no history of regular exercise habits or involvement in physical training programmes. Each subject underwent three sphygmomanometric systolic and diastolic BP measurements as well as assessment of body weight, BMI and waist and hip circumference. Total serum cholesterol, triglycerides and HDL cholesterol (enzymatic method) and plasma glucose (standard glucose oxidase method) were assayed in the fasting state from a venous blood sample. We recruited 48 subjects ( 37 males and 11 females) who displayed at least three of the five diagnostic criteria for the metabolic syndrome proposed by the Adult Treatment Panel III Report [13]: (1) waist circumference $>102 \mathrm{~cm}$ in men and $>88 \mathrm{~cm}$ in women; (2) fasting triglyceride level $>1.69 \mathrm{mmol} / \mathrm{l}$; (3) HDL cholesterol $<1.03 \mathrm{mmol} / 1$ in men and $<1.29 \mathrm{mmol} / \mathrm{l}$ in women; (4) $\mathrm{BP}>130 / 85 \mathrm{mmHg}$ and/or a history of treatment with antihypertensive drugs; and (5) fasting glucose level $\geq 6.1 \mathrm{mmol} / \mathrm{l}$. We recruited 43 additional persons ( 30 males, 13 females) as control subjects, provided that they did not display any of the abovementioned criteria for the metabolic syndrome. None of the 48 patients diagnosed as having the metabolic syndrome was under treatment with antidiabetic drugs. Eleven patients were taking antihypertensive drugs, which were withdrawn 10 days before the study. The study protocol was approved by the ethics committee of our institution. All subjects gave their written consent to the study after being informed of its nature and purpose.

Measurements Multiunit recording of efferent postganglionic muscle sympathetic nerve traffic was measured by microneurography using the peroneal nerve and following the procedure reported in detail previously $[11,12,14-$ 16]. Muscle sympathetic nerve traffic was quantified under baseline resting conditions as bursts per minute and as bursts per 100 heart beats to normalise the values for possible interindividual differences in heart rate. The quantification was extended to conditions in which baroreceptor activity was increased or reduced, with a reduction and increase, respectively, in resulting muscle sympathetic nerve traffic and increase. Changes in baroreceptor activity were obtained by i.v. infusion of vasoactive drugs [14-16]. Briefly, phenylephrine was infused incrementally through a cannula placed in an antecubital vein at doses of 0.6 and $1.2 \mu \mathrm{g} \cdot \mathrm{kg}^{-1} \cdot \mathrm{min}^{-1}$ to progressively increase mean BP (diastolic BP plus one-third of pulse pressure, beat-to-beat measurements by a finger photoplethysmographic device [Finapres 2300; Ohmeda, Englewood, CO, USA]) [14-16] and thus stimulate arterial baroreceptors. Nitroprusside was also infused incrementally at doses of 0.6 and $1.2 \mu \mathrm{g} \cdot \mathrm{kg}^{-1} \cdot \mathrm{min}^{-1}$ to progressively decrease mean BP and thus deactivate arterial baroreceptors. Both infusions were maintained for $10 \mathrm{~min}$, each step lasting $5 \mathrm{~min}$. The drug initially infused was followed by the second one after a recovery time of 45 min. Mean BP and muscle sympathetic nerve traffic were averaged for 15 baseline minutes before infusion and for 5 min of each step. Baroreceptor modulation of muscle sympathetic nerve traffic was assessed by calculating the absolute changes in sympathetic bursts per minute and the percentage changes in integrated sympathetic activity (sympathetic bursts, mean amplitude $\times$ number of bursts per minute, expressed in arbitrary units) associated with changes in mean BP induced by each dose of phenylephrine and nitroprusside [14-16]. Baroreflex sensitivity was calculated as the slope of the changes in muscle sympathetic nerve traffic divided by the changes in diastolic BP induced by vasoactive drugs (four steps) and expressed as percentage integrated activity (i.a.)/mmHg. An ECG signal and respiratory movements (pneumotachograph) were displayed together with muscle sympathetic nerve traffic on the thermal paper of an ink polygraph (Gould 3800; Gould Instruments, Cleveland, OH, USA).

Plasma norepinephrine and epinephrine were measured by HPLC [17] in blood withdrawn from an antecubital vein of the arm contralateral to that used for BP measurements. Measurements included plasma insulin concentration (RIA) in the fasting state, which allowed us to determine insulin resistance by the homeostasis model assessment (HOMA-IR); the HOMA index was calculated according to the formula: fasting plasma insulin $\times$ fasting plasma glucose/22.5 [18].

Protocol and data analysis All patients were taken to the laboratory in the morning after an overnight fast, placed supine and fitted with the i.v. cannula, the microelectrodes for muscle sympathetic nerve traffic recording and the other measuring devices. Blood samples for assaying plasma norepinephrine and epinephrine were then taken and BP was measured three times with a mercury sphygmomanometer within a 10-min interval. After $30 \mathrm{~min}$, BP, heart rate (ECG), respiration rate and muscle sympathetic nerve traffic were measured continuously over a $15-\mathrm{min}$ baseline period, the stepwise infusion of one vasoactive drug and a 45-min recovery period. This was followed by 
Table 1 Baseline demographic, anthropometric, haemodynamic and metabolic data in healthy control subjects and in patients with metabolic syndrome
Data are mean \pm SEM. ${ }^{*} p<0.05$, $* * * p<0.001$ (comparison between groups) $A U$, arbitrary units; $S$, systolic; $D$, diastolic; $M$, males; $F$, females

\begin{tabular}{lll}
\hline Variable & Control subjects $(n=43)$ & Patients with metabolic syndrome $(n=48)$ \\
\hline Sex, M/F & $30 / 13$ & $37 / 11$ \\
Age, years & $48.2 \pm 1.0$ & $49.8 \pm 1.2$ \\
BMI $\left(\mathrm{kg} / \mathrm{m}^{2}\right)$ & $24.1 \pm 0.6$ & $31.6 \pm 0.7^{* * *}$ \\
Waist circumference $(\mathrm{cm})$ & $93.6 \pm 1.5$ & $103.9 \pm 2.0^{* * *}$ \\
Sphygmo BP $(\mathrm{S} / \mathrm{D}),(\mathrm{mmHg})$ & $136.6 \pm 1.8 / 76.8 \pm 1.5$ & $149.3 \pm 1.9 / 84.9 \pm 1.9^{* * *}$ \\
Finger BP (S/D) $(\mathrm{mmHg})$ & $134.5 \pm 1.7 / 72.9 \pm 1.4$ & $147.0 \pm 2.0 / 82.6 \pm 1.8^{* * *}$ \\
Heart rate (beats/min) & $69.1 \pm 1.6$ & $72.4 \pm 1.5$ \\
Respiration rate $(\mathrm{breaths} / \mathrm{min})$ & $19.8 \pm 0.6$ & $20.2 \pm 0.6$ \\
Total cholesterol $(\mathrm{mmol} / \mathrm{l})$ & $4.84 \pm 0.1$ & $5.42 \pm 0.13^{* * *}$ \\
HDL cholesterol $(\mathrm{mmol} / \mathrm{l})$ & $1.53 \pm 0.03$ & $1.19 \pm 0.03^{*}$ \\
Triglycerides $(\mathrm{mmol} / \mathrm{l})$ & $1.15 \pm 0.07$ & $2.20 \pm 0.12^{* * *}$ \\
Fasting glucose $(\mathrm{mmol} / \mathrm{l})$ & $5.17 \pm 0.1$ & $7.42 \pm 0.2^{* * *}$ \\
Insulin $(\mu \mathrm{U} / \mathrm{ml})$ & $8.1 \pm 0.5$ & $11.9 \pm 0.8^{* * *}$ \\
HOMA index $(\mathrm{AU})$ & $1.79 \pm 0.1$ & $3.88 \pm 0.2^{* * *}$ \\
\hline
\end{tabular}

a second 15-min baseline period and finally the stepwise infusion of the second vasoactive drug. In half of the subjects, the first drug to be infused was phenylephrine, whereas in the remaining subjects it was nitroprusside.

Data were analysed by a single investigator unaware of the experimental design. Values from individual subjects were averaged for each group and expressed as mean \pm SEM. This procedure was also followed for the changes in mean BP and muscle sympathetic nerve traffic induced by each dose of vasoactive drugs. Comparisons between data obtained in the two different groups were made by two-way ANOVA. The two-tailed $t$-test for unpaired observations was used to detect between-group differences. Bonferroni correction was used to account for multiple comparisons. Spearman analysis was used to determine the correlation

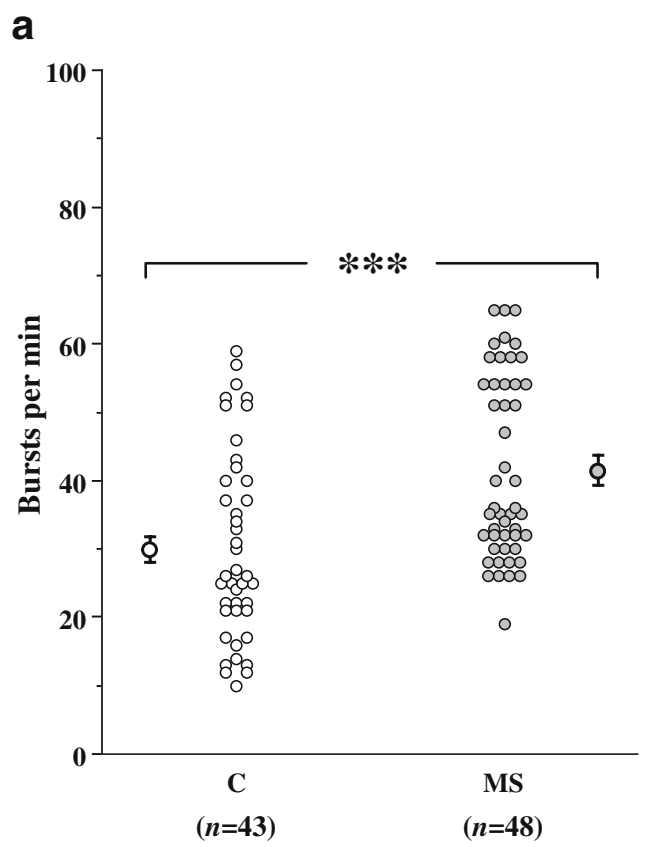

Fig. 1 Individual and mean $( \pm$ SEM) baseline values of muscle sympathetic nerve activity (MSNA), expressed as bursts per min (a) and as bursts corrected for heart rate (b) in healthy control between different variables. A value of $p<0.05$ was considered statistically significant.

\section{Results}

As shown in Table 1, the group of subjects with the metabolic syndrome and the control group were matched for age. Compared with control subjects, individuals affected by the metabolic syndrome were characterised by significantly greater values of BMI, waist circumference and sphygmomanometric and finger systolic and diastolic BPs. Serum total cholesterol, triglycerides, glucose, insulin and HOMA index values were also greater in the group with metabolic syndrome than in the control group, where-

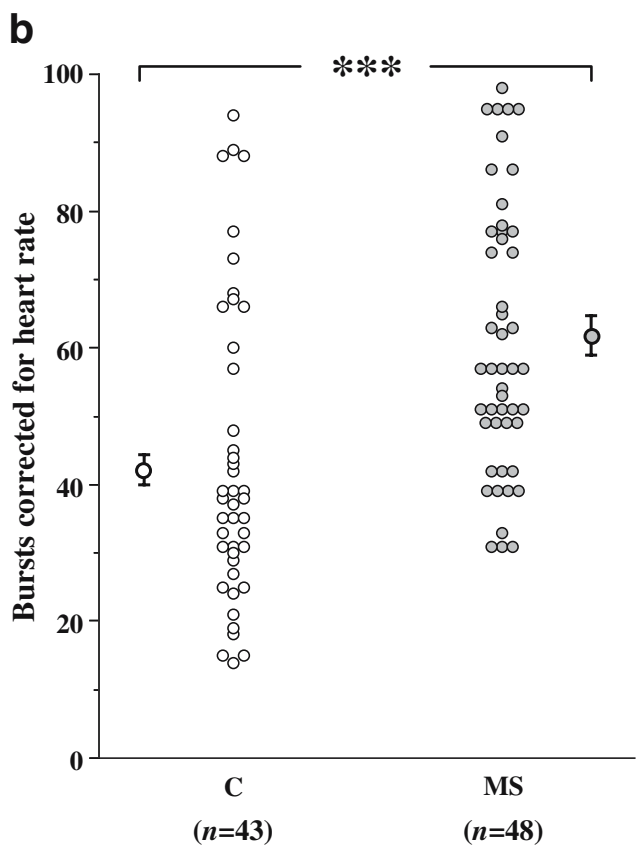

individuals $(C$, open circles $)$ and in subjects with metabolic syndrome $(M S$, closed circles). $* * * p<0.001$ (comparison between groups) 
a
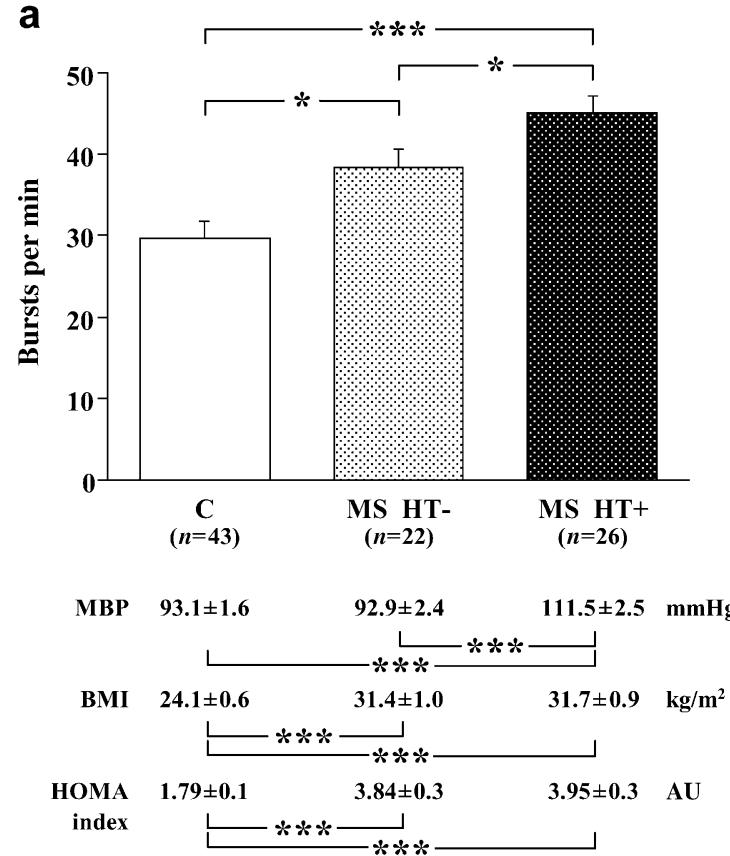

Fig. 2 Mean $( \pm$ SEM) baseline values of muscle sympathetic nerve activity, expressed as bursts per min (a) and as bursts corrected for heart rate (b) in control individuals (c) and in subjects with metabolic syndrome without (MS HT-) or with (MS HT+) hyper-

as HDL serum cholesterol values were lower. Subjects with metabolic syndrome showed greater average muscle sympathetic nerve traffic than control subjects, both when values were expressed as burst per unit time and as bursts corrected for heart rate (Fig. 1). Muscle sympathetic nerve traffic was greatest when the metabolic syndrome included

a

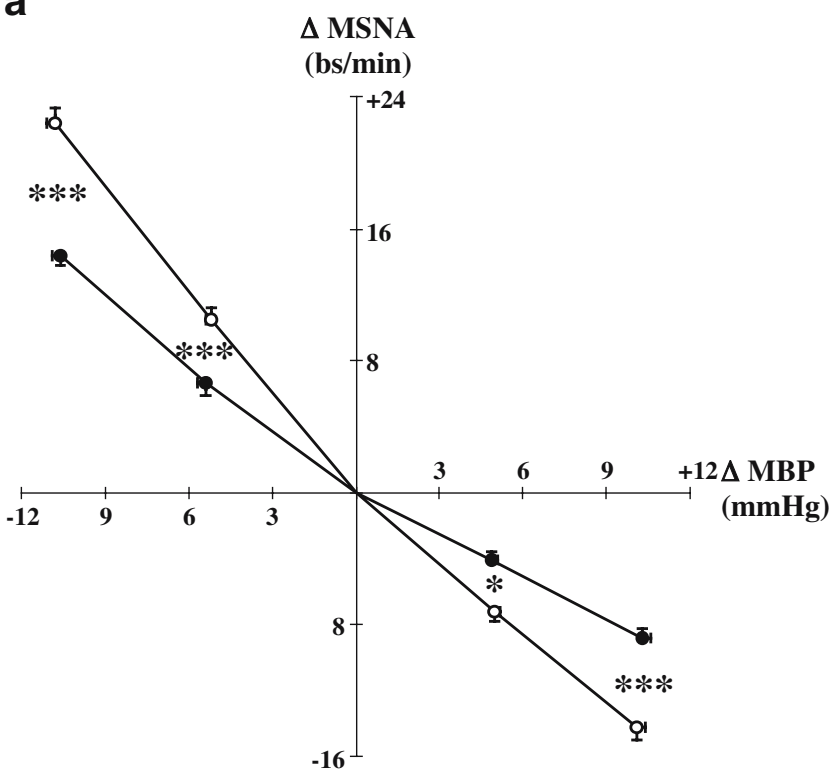

Fig. 3 Plots showing changes in muscle sympathetic nerve activity ( $\triangle$ MSNA), expressed as bursts per $\mathrm{min}$ (bs/min) (a) and as percentage integrated activity (\% i.a.) (b) accompanying stepwise increases and reductions in mean blood pressure $(\triangle \mathrm{MBP})$ induced b

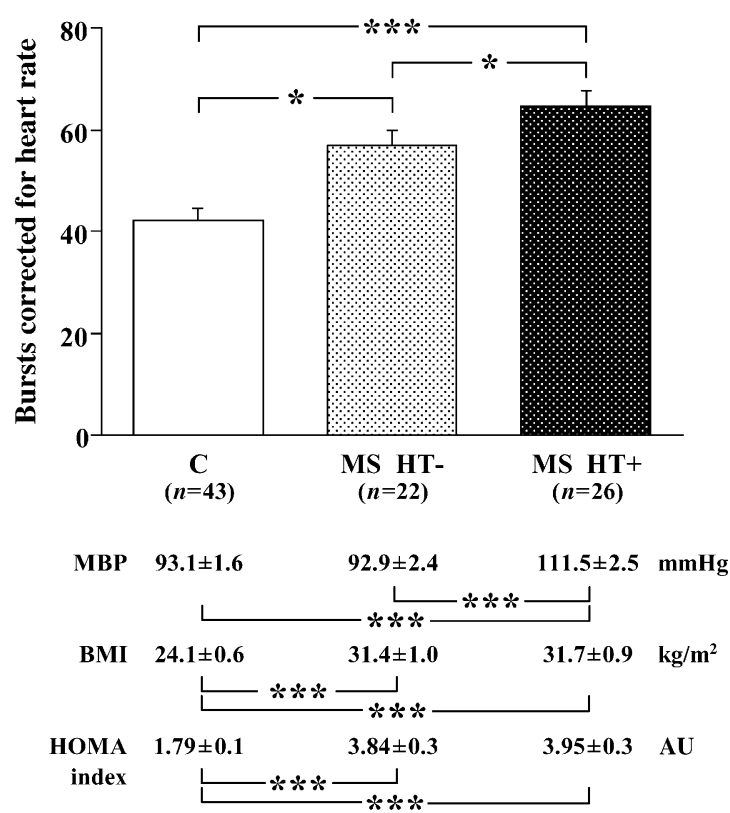

tension. Data referring to baseline mean blood pressure (MBP), BMI and HOMA index values in each group of subjects are shown below each panel. AU, arbitrary units. ${ }^{*} p<0.05, * * * p<0.001$ (comparison between groups)

hypertension as a component, but for similar increases in BMI, waist circumference and HOMA index values, sympathetic nerve traffic was also greater than in control subjects when no BP elevation was present and mean arterial pressure was similar to that of healthy subjects (Fig. 2). Muscle sympathetic nerve traffic correlated positively with

b

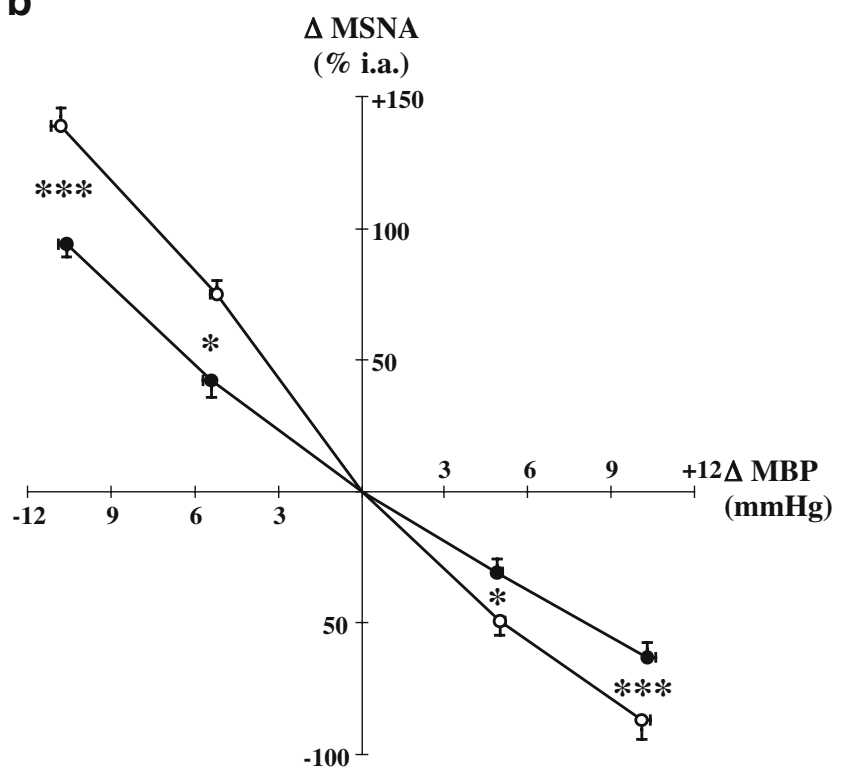

by i.v. infusion of phenylephrine and nitroprusside in control individuals (open circles) and in subjects with metabolic syndrome (closed circles). Data are expressed as mean \pm SEM. ${ }^{*} p<0.05$, $* * * p<0.001$ (comparison between groups) 
BMI $(r=0.42, p<0.001)$, waist circumference $(r=0.46, p<$ $0.001)$, HOMA index $(r=0.49, p<0.001)$ and diastolic (but not systolic) BP $(r=0.34, p<0.01)$, but it showed no correlation with serum triglycerides, total cholesterol or HDL cholesterol values $(r=0.16, r=0.11$ and $r=-0.08$ respectively, $p=\mathrm{NS}$ ). Plasma norepinephrine was slightly but not significantly greater in subjects with metabolic syndrome than in control subjects $(1.56 \pm 0.18$ vs $1.39 \pm 0.15 \mathrm{nmol} / 1$, $p=\mathrm{NS})$. This was the also case for plasma epinephrine (122.8 \pm 21.8 vs $107.5 \pm 16.37 \mathrm{pmol} / \mathrm{l}, p=\mathrm{NS})$.

Figure 3 shows that in both groups of subjects the stepwise increase in mean BP induced by phenylephrine caused a progressive decrease in muscle sympathetic nerve traffic, whereas the stepwise decrease in mean BP induced by nitroprusside had the opposite effect. The sympathoinhibitory responses to arterial baroreceptor stimulation and the sympathoexcitatory responses to arterial baroreceptor deactivation were blunted in patients with metabolic syndrome compared with control subjects, the baroreflex sensitivity values being $-9.3 \pm 0.2$ and $-14.6 \pm 0.4 \%$ i.a. $/ \mathrm{mmHg}$, respectively ( $p<0.01$ between groups). There was no difference in baroreflex-muscle sympathetic nerve traffic sensitivity between normotensive and hypertensive patients with metabolic syndrome $(-9.6 \pm 0.3$ vs $9.1 \pm 0.4 \%$ i.a./ $\mathrm{mmHg}$ respectively, $p=\mathrm{NS}$ ). Baseline muscle sympathetic nerve traffic values were significantly $(p<0.001)$ and inversely related to baroreflex sensitivity both in subjects with metabolic syndrome and in control subjects. In the former group the correlation coefficient was higher ( $r=$ -0.44 vs $r=-0.35$ ) but the difference did not achieve statistical significance.

\section{Discussion}

The present study provides evidence that in subjects with metabolic syndrome sympathetic activity is increased independently of the presence of cardiovascular drugs (such as calcium antagonists and diuretics) which trigger sympathetic activation [11] per se. It also provides evidence that the sympathetic activation is not limited to individuals in whom the metabolic syndrome is accompanied by hypertension, i.e. a condition in which sympathetic activity is increased regardless of the presence of increased body weight or other metabolic abnormalities [11, 16, 19, 20], because sympathetic nerve traffic was also greater than in control subjects when the metabolic syndrome was associated with normal BP values, and thus its diagnosis depended on criteria other than the BP elevation. This allows us to conclude that a hyperadrenergic state represents an intrinsic feature of this condition.

Our study also provides information on the mechanisms that may be responsible for the sympathetic hyperactivity characterising the metabolic syndrome. In subjects with metabolic syndrome, muscle sympathetic nerve traffic exhibited a positive relationship with the HOMA index, a variable reflecting insulin resistance. This supports the dependence of the adrenergic activation on the hyperinsulinaemia that follows the insulin resistance state, given the well-known ability of insulin to stimulate sympathetic nerve activity, particularly to skeletal muscle tissues $[6,7$, 21-23]. However, muscle sympathetic nerve traffic also showed a positive significant relationship with BMI, the increase of which may stimulate sympathetic drive via (1) the central influence of greater circulating levels of leptin $[24,25]$ and (2) the reflex influence of chemoreceptor activation, as shown in patients with sleep apnoea [26, 27], which is more prevalent in patients with the metabolic syndrome [28]. Furthermore, in subjects with metabolic syndrome there was an attenuation of the sensitivity of baroreceptor sympathetic control, which suggests that impairment of the ability of the baroreflex to restrain adrenergic tone is also involved. Thus, the hyperadrenergic state of the metabolic syndrome may be mechanistically complex because of the involvement of metabolic, central and reflex factors. It is possible that the sympathetic hyperactivity represents the earliest abnormality of the disease, given the evidence that sympathetic stimulation can cause insulin resistance by constricting skeletal muscle vessels and increasing the distance insulin has to travel to allow glucose to cross cell membranes [29-31]. It should be emphasised, however, that reduction in body weight and insulin resistance brought about by low caloric intake leads to sympathetic deactivation [15], which suggests that, on the contrary, metabolic factors play the initial role. The observational nature of our study does not allow this 'chicken and egg' question to be answered conclusively.

Other results of our study deserve to be discussed. First, data obtained by spectral analysis of heart rate suggest that in the metabolic syndrome there is an increased sympathetic discharge to the heart [32]. Although spectral analysis represents an indirect and not entirely specific sympathetic marker [11, 33, 34], this finding suggests that the sympathetic hyperactivity of the metabolic syndrome is not confined to the cardiac district but involves regional circulations as well. Secondly, because components of the metabolic syndrome such as visceral obesity and hypertension both have sympathostimulating effects, the question arises whether, in this condition, their association leads to greater overall sympathetic activation. Our present finding that inclusion of hypertension in the metabolic syndrome triggered a sympathetic activation that was greater in magnitude than that found when high blood pressure was excluded suggests that this may be indeed the case. This is in line with our previous observation that the sympathetic hyperactivity characterising hypertension, obesity and heart failure undergoes summation when these conditions coexist in the same patient [35]. It remains to be seen, however, whether in the metabolic syndrome the association of obesity or hypertension with alterations in lipid profile makes the sympathostimulating effect different from that of either condition alone. The finding in the present study that sympathetic nerve traffic did not correlate with serum triglyceride and LDL cholesterol values scores against this. The third result worthy of discussion is that plasma norepinephrine was slightly but not significantly greater in subjects with metabolic syndrome than in control subjects. This strengthens the evidence that in a variety of conditions the 
plasma level of the main adrenergic neurotransmitter represents an insensitive marker of sympathetic drive [11, 14, 36-38].

Our results have pathophysiological and clinical implications. First, because the sympathetic nervous system exerts a trophic influence on cardiac myocytes [39], the adrenergic hyperactivity characterising patients with metabolic syndrome may well explain why, for similar BP values, patients with metabolic syndrome have been found to display not only increased urinary protein excretion [40] but also a left ventricular mass index greater than that in subjects without this condition [41]. Secondly, the increase in neural sympathetic outflow reported in our patients may be, at least in part, responsible for the finding that the metabolic syndrome is characterised by a dysfunction in $\beta_{3}$ adrenoreceptor activity at the level of adipose tissue [42]. Finally, the evidence that the metabolic syndrome is characterised by an augmented sympathetic drive supports the recommendation to treat this condition mainly by body weight reduction and an increase in physical activity [5, 43]. This is because these non-pharmacological interventions have been documented to exert marked and longlasting sympathoinhibitory effects $[15,44]$.

\section{References}

1. Isomaa B, Almgren P, Tuomi $\mathrm{T}$ et al (2001) Cardiovascular morbidity and mortality associated with the metabolic syndrome. Diabetes Care 24:683-689

2. Lakka HM, Laaksonen DE, Lakka TA et al (2002) The metabolic syndrome and total and cardiovascular disease mortality in middle-aged men. JAMA 288:2709-2716

3. Isomaa B, Henricsson M, Almgren P, Tuomi T, Taskinen MR, Groop L (2001) The metabolic syndrome influences the risk of chronic complications in patients with type II diabetes. Diabetologia 44:1148-1154

4. Heine RJ, Dekker JM (2002) Beyond postprandial hyperglycaemia: metabolic factors associated with cardiovascular disease. Diabetologia 45:461-475

5. Scott MG, Brewer HB, Cleeman JI et al (2004) Definition of metabolic syndrome. Report of the National Heart, Lung and Blood Institute/American Heart Association Conference on scientific issues related to definition. Circulation 109:433-438

6. Reaven GM, Lithell H, Landsberg L (1996) Hypertension and associated metabolic abnormalities: the role of insulin resistance and the sympathoadrenal system. N Engl J Med 334:374381

7. Landsberg L (2001) Insulin-mediated sympathetic stimulation: role in the pathogenesis of obesity-related hypertension. J Hypertens 19:523-528

8. Huggett RJ, Burns J, Mackintosh AF, Mary D (2004) Sympathetic neural activation in nondiabetic metabolic syndrome and its further augmentation by hypertension. Hypertension 44:847-852

9. Suter PM, Vetter W (1995) Metabolic effects of antihypertensive drugs. J Hypertens 13:S11-S17

10. Neutel JM (1996) Metabolic manifestations of low dose diuretics. Am J Med 101:71S-82S

11. Grassi G, Esler MD (1999) How to assess sympathetic activity in humans. J Hypertens 17:719-734

12. Wallin BG, Fagius J (1988) Peripheral sympathetic neural activity in conscious humans. Annu Rev Physiol 50:565-576
13. Third Report of the National Cholesterol Education Program (NCEP) Expert Panel on Detection, Evaluation, and Treatment of high Blood Cholesterol in Adults (Adult Treatment Panel III) Final Report (2002) Circulation 106:3143-3421

14. Grassi G, Seravalle G, Cattaneo BM et al (1995) Sympathetic activation in obese normotensive subjects. Hypertension 25: 560-563

15. Grassi G, Seravalle G, Colombo M et al (1998) Body weight reduction, sympathetic nerve traffic, and arterial baroreflex in obese normotensive humans. Circulation 97:2037-2042

16. Grassi G, Cattaneo BM, Seravalle G et al (1998) Baroreflex control of sympathetic nerve activity in essential and secondary hypertension. Hypertension 31:68-72

17. Hjemdahl P, Daleskog M, Kahan T (1979) Determination of plasma catecholamines by high performance liquid chromatography with electrochemical detection: comparison with a radioenzymatic method. Life Sci 25:131-138

18. Matthews DR, Hosker JP, Rudenski AS et al (1985) Homeostasis model assessment: insulin resistance and beta-cell function from fasting plasma glucose and insulin concentrations in man. Diabetologia 28:412-419

19. Schlaich MP, Kaye DM, Lambert E et al (2003) Relation between cardiac sympathetic activity and hypertensive left ventricular hypertrophy. Circulation 108:560-565

20. Schlaich MP, Lambert E, Kaye DM et al (2004) Sympathetic augmentation in hypertension: role of nerve firing, norepinephrine reuptake, and angiotensin neuromodulation. Hypertension 43:169-175

21. Rowe JW, Young JB, Minaker KL, Stevens AL, Pallotta J, Landsberg L (1981) Effect of insulin and glucose infusions on sympathetic nervous system activity in normal man. Diabetes 30:219-225

22. Anderson EA, Balon TW, Hoffmann RP et al (1992) Insulin increases sympathetic activity but not blood pressure in borderline hypertensive humans. Hypertension 19:62-69

23. Berne C, Fagius J, Pollare T, Hjemdhal P (1992) The sympathetic response to euglycaemic hyperinsulinemia. Evidence from microelectrode nerve recordings in healthy subjects. Diabetologia 35:873-879

24. Correia ML, Haynes WG (2004) Leptin, obesity and cardiovascular disease. Curr Opin Nephrol Hypertens 13:215-223

25. Grassi G (2004) Leptin, sympathetic nervous system and baroreflex function. Curr Hypertens Rep 6:236-240

26. Somers VK, Dyken ME, Clary MP et al (1995) Sympathetic neural mechanisms in obstructive sleep apnea. J Clin Invest 96:1897-1904

27. Narkiewicz K, Van de Borne PJH, Cooley RL et al (1998) Sympathetic activity in obese subjects with and without obstructive sleep apnea. Circulation 98:772-776

28. Coughlin SR, Mawdsley L, Mugarza JA, Calverley PM, Wilding JP (2004) Obstructive sleep apnoea is independently associated with an increased prevalence of metabolic syndrome. Eur Heart J 25:709-711

29. Jamerson KA, Julius S, Gudbrandsson T et al (1993) Reflex sympathetic activation induces acute insulin resistance in the human forearm. Hypertension 21:618-623

30. Julius S, Jamerson K (1994) Sympathetics, insulin resistance and coronary risk in hypertension: 'chicken-and-egg' question. J Hypertens 12:495-502

31. Baron AD, Brechtel-Hook G, Johnson A et al (1996) Effect of perfusion rate on the time course of insulin-mediated skeletal muscle glucose uptake. Am J Physiol 271:E1067-E1072

32. Brunner EJ, Hemingway H, Walker BR et al (2002) Adrenocortical autonomic and inflammatory causes of the metabolic syndrome. Circulation 106:2659-2665

33. Kingwell BA, Thompson JM, Kaye DM et al (1994) Heart rate spectral analysis, cardiac norepinephrine spillover and muscle sympathetic nerve activity during human sympathetic nervous activation and failure. Circulation 90:234-240 
34. Eckberg DL (1997) Sympathovagal balance: a critical appraisal. Circulation 96:3224-3232

35. Grassi G, Seravalle G, Quarti-Trevano F et al (2003) Effects of hypertension and obesity on the sympathetic activation of heart failure patients. Hypertension 42:873-877

36. Folkow B, Di Bona GF, Hjemdahl P et al (1983) Measurements of plasma norepinephrine concentrations in human primary hypertension. Hypertension 5:399-403

37. Meredith IT, Eisenhofer G, Lambert GW et al (1992) Plasma norepinephrine responses to head-up tilt are misleading in autonomic failure. Hypertension 19:628-633

38. Grassi G, Bolla GB, Seravalle G et al (1997) Comparison between reproducibility and sensitivity of muscle sympathetic nerve traffic and plasma noradrenaline in man. Clin Sci 92: 285-289

39. Deng XF, Rokosh DG, Simpson PC (2000) Autonomous and growth factor-induced hypertrophy in cultured neonatal mouse cardiac myocytes. Comparison with rat. Circ Res 87:781-788
40. Palaniappan L, Carnethon M, Fortmann SP (2003) Association between microalbuminuria and the metabolic syndrome: NHANES III. Am J Hypertens 16:952-958

41. Cuspidi C, Meani S, Fusi V et al (2004) Metabolic syndrome and target organ damage in untreated essential hypertension. J Hypertens 22:1991-1998

42. Hoffstedt J, Wahrenberg H, Thorne A, Lonnqvist F (1996) The metabolic syndrome is related to $\beta 3$-adrenoceptor sensitivity in visceral adipose tissue. Diabetologia 39:838-844

43. Grundy SM, Hansen B, Smith SC et al (2004) Clinical management of metabolic syndrome. Report of the American Heart Association/National Heart, Lung and Blood Institute/ American Diabetes Association Conference on Scientific issues related to management. Circulation 109:551-556

44. Grassi G, Seravalle G, Calhoun DA, Mancia G (1994) Physical training and baroreceptor control of sympathetic nerve activity in humans. Hypertension 23:294-301 